\title{
Prevention of Cardiovascular Disease by Dietary n-3 Fatty Acid
}

\author{
B. Raghu.
}

Associate Professor, International M edical School, M anagement and Science University, Shah Alam 40100, Malaysia.

\section{ABSTRACT}

Cardiovascular disease (CVD) is a main cause of morbidity and mortality worldwide. Its prevention through a healthy lifestyle and appropriate diet is important. Omega-3 polyunsaturated fatty acids ( $n-3$ PUFA) therapy has shown promise in both primary and secondary prevention of CVD. This commentary reviews the nutritional role of n-3 PUFA, including its metabolism and physiological role, comparison to n-6 series PUFA, as well as complications due to deficiency. Clinical use of n-3 PUFA for the prevention and treatment of CVD, recommended intake and potential adverse effects will also be examined. The available scientific evidence suggests that its supplementation and clinical use ranging from 0.4 to $7 \mathrm{~g} /$ day can provide tangible benefits. However, further studies are required to determine optimal dosing and the relative ratio of docosahexaenoic acid and eicosapentaenoic acid that provides maximal cardio-protection and treatment of CVD.

KEY WORDS: Alpha-Linolenic Acid, Docosahexaenoic Acid, Eicosapentaenoic Acid, Cardiovascular Disease, Fish Oil, Polyunsaturated Fatty Acids.

Address for correspondence: B. Raghu, Associate Professor, International Medical School, M anagement and Science University, Shah Alam 40100, Malaysia.E-Mail: vasuraghu@ hotmail.com

\begin{tabular}{|c|c|}
\hline \multicolumn{2}{|r|}{ Online Access and Article Informtaion } \\
\hline 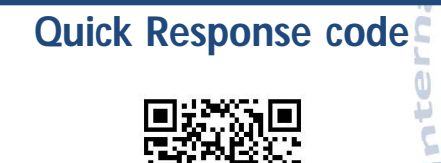 & $\begin{array}{c}\text { International Journal of Integrative Medical Sciences } \\
\text { www.imedsciences.com }\end{array}$ \\
\hline Dol: $10.16965 /$ ijims.2016.124 & $\begin{array}{ll}\text { Received: 02-06-2016 } & \text { Accepted: 26-08-2016 } \\
\text { Reviewed: 02-06-2016 } & \text { Published: 06-10-2016 }\end{array}$ \\
\hline Source of Funding: Self & Conflicts of interest: None \\
\hline
\end{tabular}

\section{BACKGROUND}

Cardiovascular diseases (CVD) are a group of disorders of the heart and blood vessels, and include coronary heart disease (CHD), cerebrovascular disease (stroke), raised blood pressure (hypertension), peripheral artery disease, rheumatic heart disease, congenital heart disease and heart failure. According to the World Health Organization, CVD are the number one cause of death globally and claim 17 million lives each year. By 2030, almost 24 million people will die from CVD, mainly from heart disease and stroke. These are projected to remain the single leading causes of death [1]. In the United States, CVD account for more than one-third $(34.3 \%)$ of deaths annually, and responsible for nearly 3 million Americans reporting disability. The costs of CVD are also staggering. In 2010, the total cost including health care services, medications and lost productivity, is estimated to be over $\$ 503$ billion in the United States [2]. Similarly, the National Heart Foundation of Australia reported that CVD are the leading cause of mortality and morbidity in Australia, killing one person nearly every 10 minutes [3]. Despite improvements over the last few decades, CVD remain as the second largest disease burden to our society after cancers. As the population ages, the economic impact of CVD on the health care system will become even greater. Tobacco smoking, an unhealthy diet, physical inactivity and high alcohol consumption increase the risk of CVD. Indeed, behavioral and dietary risk factors are responsible for about 
$80 \%$ of coronary heart disease and cerebrovascular disease.

Interestingly, both the incidence and mortality rate of CVD are much lower in Japan than other countries [4], which may be attributed to the high consumption of fish/seafood by the Japanese population [5]. The fact that fish is abundant in omega-3 polyunsaturated fatty acids has opened an effective venue to the prevention and treatment of this disease by either dietary modifications or pharmacological supplementation.

Polyunsaturated fatty acids: Fat is one of the energy sources, and is indispensable for physiological functions such as delivery of lipidsoluble vitamins, materials of biomembranes and bioactive substances. Triglycerides are the most common fat in our diet. The term "fat" refers to triacylglycerols, which are composed of three fatty acid molecules attached to a glycerol backbone. Fatty acids consist of a hydrocarbon chain and a carboxyl group. Depending on the structural difference, they are categorized into two major groups, saturated fatty acids and unsaturated fatty acids, with or without one or more double bonds. Saturated fatty acids, the substantial components in animal fat, have only single bonds in the hydrocarbon chain.

Unsaturated fatty acids have one or more double bonds in the hydrocarbon chain. Furthermore, the unsaturated fatty acids are classified into two groups, monounsaturated fatty acids and polyunsaturated fatty acids (PUFA). There are two main types of bioactive PUFA, namely, the omega- $6(n-6)$ series, and the omega- $3(n-3)$ series. The $n-6$ series comprise linoleic acid (LA, 18:2 n-6), gamma-linolenic acid (GLA, 18:3 n6), arachidonic acid (AA, 20:4 $n-6$ ) and others. The $n-3$ series include alpha-linolenic acid (ALA, 18:3 n-3), eicosapentaenoic acid (EPA, 20:5 n3), docosapentaenoic acid (DPA, 22:5n-3) and docosahexaenoic acid (DHA, 22:6n-3). Both $n$ 3 PUFA and n-6 PUFA are important constituents of all cell membranes. Because PUFA such as $L A$ and ALA cannot be synthesized in the human body, that is, unable to desaturate $\mathrm{C}-\mathrm{C}$ bonds of the $n-3$ and $n-6$ carbons involved in the hydrocarbon chain of fatty acids, these PUFA must be obtained from diet and thus are called essential fatty acids. Common sources of $n-3$ PUFA include fish oils (EPA and DHA) and some plant oils such as canola and flaxseed oils (ALA). While an overall reduction of fat intake is advisable especially in Western countries, more emphasis has been placed on the relative proportion of individual fatty acids.

Scope of review: In the literature, observational and experimental studies especially clinical trials have found n-3 PUFA as important in the protection against CVD. The most compelling evidence of its cardiovascular benefits came from studies of patients following myocardial infarction, and most recently, in patients with heart failure. Besides, the level of n-3 PUFA may act as both prognostic and diagnostic utility in CVD risk assessment.

In this commentary, we consider the scientific evidence showing the potential benefits of $n-3$ PUFA, particularly EPA and DHA in primary and secondary cardiovascular prevention. Our review will include the metabolism of n-3 PUFA, its physiological role, comparison to n-6 PUFA, as well as complications due to deficiency. Potential clinical use for CVD risk management will then be examined, including the recommended dosage that can provide maximal cardio-protection. However, detailed physiology and biological mechanisms underlying the benefits of the $n-3$ series PUFA are beyond the scope of this review.

\section{METABOLISM AND PHYSIOLOGICAL ROLE OF PUFA}

Metabolism: Humans can in theory synthesize longer $n-6$ and $n-3$ FA from the essential fatty acids $L A$ and $A L A$, respectively, through a series of desaturation (addition of a double bond) and elongation (addition of two carbon atoms) reactions. However, these activities are known to be weak and the human body can only convert small amounts of ALA into EPA (about 5\%) and DHA $(<0.5 \%) .{ }^{6}$ The $n-6$ PUFA and n-3 PUFA are metabolized through different metabolic pathways to produce bioactive substances called eicosanoids. Eicosanoids, such as prostaglandins, leukotrienes, and thromboxanes, have functions of smooth muscle contraction, inflammatory reaction, and platelet aggregation. The eicosanoids from each series work as 
autacoids and have similar functions but different in potency. Eicosanoids from the n-3 series work mildly showing antagonizing effect against the strong function of eicosanoids from the $n-6$ series. For details on the biochemistry and metabolism of PUFA, the readers are referred to a recent comprehensive review [7].

Physiological role: Both $n-6$ and n-3 PUFA are important structural components of cell membranes. When incorporated into phospholipids, they affect cell membrane properties such as fluidity, flexibility, permeability and the activity of membrane bound enzymes [8]. In many cases, $n-3$ and $n-6$ series can compensate each other's function in ameliorating pathological conditions, such as growth retardation. In other situations, the biological activity of the $n-3$ series is more specific. DHA is incorporated into retinal cell membranes and is required for the normal development and function of the retina, suggesting its key role in vision. The phospholipids of the brain's gray matter contain high proportions of DHA and AA, so that they are crucial to central nervous system function [9]. It appears that n-3 PUFA can reduce inflammation in the brain and play a specific role in brain development and regeneration of nerve cells [10]. Indeed, animal studies have shown that depletion of DHA in the brain can result in learning deficits. It is unclear how DHA affects brain function, but changes in DHA content of neuronal cell membranes can alter the function of ion channels or membraneassociated receptors. DHA influences signaling events which are vital for neuronal survival and differentiation [7]. Moreover, $n-6$ and n-3 PUFA can modulate the expression of a number of genes, including those involved with fatty acid metabolism and inflammation. Although the mechanisms require further clarification, $\mathrm{n}-6$ and n-3 PUFA may regulate gene expression by interacting with specific transcription factors [11].

The need for n-3 PUFA during pregnancy for fetal neurological growth and development is well recognized. The International Society for the Study of Fatty Acids and Lipids has recommended an intake of $200 \mathrm{mg}$ DHA during pregnancy and lactation [12]. Actually, the benefits of n-3 PUFA may well extend to cerebral circulation, whose defects are associated with decreased cognitive ability and dementia, which are common complaints of the old age [13].

Deficiency: Clinical signs of essential FA deficiency include a dry scaly rash, decreased growth in infants and children, increased susceptibility to infection, and poor wound healing [14]. Because n-3 PUFA are highly concentrated in the brain, they are important for cognitive and behavioral function. In fact, infants who do not get enough n-3 PUFA from their mothers during pregnancy are at risk of developing vision and nerve problems. Previous studies have suggested that a dietary insufficiency of n-3 PUFA may contribute to a variety of disorders including fatigue, itchy skin, brittle hair and nails. Other symptoms of deficiency are frequent colds, poor concentration/memory and lack of physical endurance. Recently, researchers found that reduced levels of n-3 PUFA have deleterious consequences on synaptic functions and emotional behaviors such as depression and anxiety [15].

\section{THE n"6 TO n"3 RATIO}

Clinical studies have indicated that the ingested ratio of $n-6$ to $n-3$ fatty acids (especially LA versus $A L A)$ is important to maintain cardiovascular health $[16,17]$. As mentioned in Section 2 above, both $n-3$ and $n-6$ PUFA are essential and they compete for the same metabolic enzymes. Therefore, their intake ratio will significantly influence the ratio of the ensuing eicosanoids (hormones), (eg, prostaglandins, leukotrienes, thromboxanes) and will alter the body's metabolic function [18]. M etabolites of $n-6$ can be more inflammatory (especially $A A$ ) than those of $n-3$. This may necessitate $n-3$ and $n-6$ PUFA be consumed in a balanced manner. It has been observed that people nowadays tend to take excessive n- 6 fatty acids and lack of n-3 fatty acids in their diet. Consequently, cells must adapt to this surplus $n-6$ and dietary deficiency of n-3 PUFA. Because both series regulate a number of transcription factors and interact with nuclear receptors which influence inflammatory responses and lipid metabolism, an imbalance $n-6: n-3$ ratio may result in altered gene regulation and expression in downstream pathways, resulting in altered 
protein expression and activity that can negatively affect cell membrane composition and fluidity and organ function [19].

It has also been hypothesized that a diet rich in n-6 PUFA content increases the susceptibility of low-density lipoprotein particles to oxidation, which in turn leads to atherosclerosis and CVD. However, the detrimental effects from higher intake of n-6 PUFA have not been fully supported by evidence in humans [20]. For example, a large follow-up study in the United States found that the $n-6: n-3$ ratio was not linked to the risk of heart disease because both of them were beneficial [21].

In view of the potential antagonizing effect of each eicosanoids group derived from the $n-6$ and the $n-3$ series in the presence of conflicting scientific evidence, there have been controversies regarding the recommended adequate level of $n-6$ and $n-3$ PUFA intakes and their intake ratio. The recommended ratio of $n$ $6: n-3$ PUFA has ranged between 4 to 10 in the United States and European countries, and is about 4 in Japan based on the 2010 edition of the Dietary Reference Intakes for Japanese [22]. Recent estimations of PUFA consumption in the food chain in Europe and the United States showed that the ratio is still much higher than that recommended. It has been reported that healthy subjects fed with a typical Swedish diet had a $n-6: n-3$ ratio measured in the serum of 4.72, whereas individuals who ate a Mediterranean-style diet resulted in a serum n6:n-3 ratio of 2.6 [23]. In general, it is difficult to resolve the issue of an optimal $n-6: n-3$ ratio universally because their intake are easily influenced by diet culture and environmental factors which vary greatly between countries. For example, the traditional Japanese diet contains 8 to 15 times more n-3 PUFA than does a typical non-Japanese diet [24].

Instead of the n-6:n-3 ratio, another marker of CVD risk is the $n-3$ index, on the basis of plasma concentrations of $n-3$ PUFA being a predictor of sudden cardiac death [25]. This index reflects the proportion of $n-3$ PUFA in the membrane of red blood cells, with below $4 \%$ indicative of coronary artery disease whereas levels exceeding $8 \%$ give the lowest risk of CVD events. It may thus be used to identify individuals requiring $n-3$ PUFA supplementation and/or dietary modification [26].

\section{CLINICAL USE FOR CARDIOVASCULAR DISEASE PREVENTION}

The link between n-3 PUFA and CVD risk reduction has been extensively studied during the past three decades. Research has shown that $n-3$ PUFA can prevent fatal arrhythmias (responsible for sudden cardiac death), lower blood pressure and plasma triglyceride levels, improve autonomic function and reduce the growth rate of atherosclerotic plaque, among others, as summarized by a recent review [27], although the relative effects of DHA and EPA remain uncertain. n-3 PUFA supplementation is often used in the secondary prevention of fatal and nonfatal myocardial infarction [28]. Its therapeutic actions have been recently extended to the prevention and treatment of heart failure. A large prospective cohort study of nearly 60,000 Japanese found an inverse association between fish and n-3 PUFA consumption and CVD mortality, especially for heart failure [29]. The finding raised the possibility that n-3 PUFA supplementation and pharmacological doses may be needed in different stages of heart failure.

In relation to clinical use, evidence from three systematic reviews supports a neutral effect on CVD mortality for n-3 PUFA intake of $400 \mathrm{mg}$ up to $7000 \mathrm{mg}$ per day [30-32], whereas no scientific data was available beyond $7000 \mathrm{mg} /$ day. Other systematic reviews focused on surrogate markers of CVD such as lipid profiles, the majority of which found a protective effect of $n-3$ PUFA on triglycerides and a neutral or small adverse effect on total cholesterol, low density lipoprotein cholesterol and high density lipoprotein cholesterol [33-39]. M oreover, there is little evidence on vegetable origin n-3 PUFA in relation to CVD. Although a wide range of adult populations predominantly from America and Europe were considered in these reviews, it should be cautioned that some of the systematic reviews included subjects who had already experienced a CVD event and were secondary prevention trials. As such, the results must be interpreted with caution because these studies could not be removed from the 
systematic review or meta-analysis. In addition to different study designs and populations, the variability in results across clinical trials may be due to confounding factors such as the patients' baseline diet, the types and doses of EPA and DHA given, the duration of treatment, and patient compliance [6].

The mechanisms by which n-3 PUFA reduce CVD morbidity and mortality are manifold [40]. The activities have been extensively investigated in human intervention trials and in vitro mechanistic studies. A detailed discussion of the potential mechanisms is beyond the scope here. There appears to be two main mechanisms. Firstly, the suppression of cardiac arrhythmias, by (a) directly inhibiting voltage-gated sodium channels and L-type calcium channels, resulting in a longer relative refractory period and in an increased voltage required for membrane depolarization, which in turn reduces heart rate; (b) improving left ventricular efficiency and reducing blood pressure, which indirectly contribute to lowering the heart rate. Secondly, n-3 PUFA can reduce atherogenesis, occurring through the modulation of specific atherothrombotic risk factors, decreased platelet aggregation, reduced plasma triglycerides and blood pressure, and above all, the regulation of systemic and local inflammation underlying plaque inception, progression and instability [27,41-49]. For patients with heart failure, $n-3$ PUFA may also invoke stimulation of peroxisome proliferator-activated receptor alpha receptors, improved mitochondrial function and down-regulation of anti-inflammatory pathway [42]. The readers are referred to recent reviews for more details $[19,41]$.

\section{RECOM MENDED INTAKE AND SIDE EFFECT}

Several guidelines have been issued by scientific bodies. For example, the American Heart Association (AHA) had released a scientific statement on the effects of n-3 PUFA on heart function (including antiarrhythmic effects), hemodynamics (cardiac mechanics) and arterial endothelial function [43]. For patients with documented CHD, the AHA currently endorses the use of n-3 PUFA at a dose of approximately $1000 \mathrm{mg} /$ day of combined DHA and EPA, either in the form of fatty fish or fish oil supplements (in capsules or liquid form). For patients who need to lower triglycerides, 2000 to $4000 \mathrm{mg}$ should be taken as capsules under a physician's care. The AHA has further recommended everyone to "eat a variety of (preferably oily) fish at least twice a week. Include oils and foods rich in ALA (flaxseed, canola and soybean oils; flaxseed and walnuts)" [43]. The National Heart Foundation of Australia similarly recommends the consumption of $500 \mathrm{mg}$ of combined DHA and EPA every day through a combination of two to three $150 \mathrm{~g}$ serves of oily fish per week, fish oil supplements (capsules or liquid) and food and drinks enriched with marine n-3 PUFA, in addition to at least $2 \mathrm{~g}$ of ALA intake per day [44]. However, the Australian National and Medical Research Council estimated that consuming at least $90 \mathrm{mg} /$ day for women and $160 \mathrm{mg} /$ day for men of $\mathrm{n}-3$ PUFA (marine source) and $800 \mathrm{mg} /$ day for women and $1300 \mathrm{mg} /$ day for men of ALA should be adequate for good health. To prevent chronic disease, target intake levels for combined DHA, EPA and DPA are $430 \mathrm{mg} /$ day for women and $610 \mathrm{mg} /$ day for men, with upper limit being set at $3000 \mathrm{mg} /$ day [45]. In addition to over the counter supplements (fish oils), standardized capsulated concentrate of EPA and DHA as a prescription drug is readily available in the market.

The International Society for the Study of Fatty Acids and Lipids recommends an intake of ALA of $0.7 \%$ of energy, and a minimum intake of 500 $\mathrm{mg} /$ day of combined EPA and DHA [12]. In spite of the global consensus on the $500 \mathrm{mg}$ intake level for healthy adults set by various government organizations and professional bodies, and a separate recommendation of 200 mg DHA for pregnant and lactating women [12], with the exception of Japan and a few isolated places, most people in the world are not consuming sufficient n-3 PUFA for optimal health. For example, the average n-3 PUFA intake of Australians has been estimated at $246 \mathrm{mg} /$ day, well below the Japanese median intake of $810 \mathrm{mg} /$ day in 2003 [5].

It is notew orthy that DHA and EPA differ in their ability to promote various effects of $n-3$ PUFA. Indeed, the overlapping and separate mechanisms by which DHA and EPA prevent and reverse CVD are still unclear [19]. The optimal 
doses and ratios of DHA to EPA are difficult to determine, as both are present in most fish especially oily fish, generally in a 2:1 ratio [46].

As far as safety is concerned, unlike cardiac pharmaceuticals, n-3 PUFA supplementations are well tolerated. High doses of $n-3$ PUFA may in theory increase the bleeding time by inhibiting the AA pathway. Clinically, it does not pose significantly increased risk of bleeding for patients undergoing coronary artery bypass grafting, carotid endarterectomy or femoral artery catheterization, even at high doses combined with antiplatelet therapy or warfarin [27]. The most commonly observed side effects are nausea, gastrointestinal symptoms, fish-scented halitosis and dysgeusia, which are not considered as major health hazards [42]. There is also some concern about ingestion of mercury when fishes are eaten, particularly for species like shark and swordfish. Nevertheless, commonly consumed oily fish such as salmon, tuna, mackerel, trout and sardines contain very low levels of mercury, while purified fish oils in pharmaceutical grade capsules typically have negligible amounts [47].

\section{SUMM ARY AND PERSPECTIVES}

CVD will continue to be the leading cause of mortality worldwide due to the escalating aging populations coupled with the increasing incidence of obesity and type 2 diabetes, which share many of the emerging risk factors for CVD. The $n-3$ series PUFA play a crucial role in brain function as well as normal growth and development. Fish, particularly fatty fish like salmon, mackerel, herring, lake trout, sardines and tuna, is a good source of n-3 PUFA. The AHA Dietary Guidelines currently recommend eating fish at least two times (two servings) a week. Each serving is 3.5 ounce cooked, or about $3 / 4$ cup of flaked fish [48]. This amount is equivalent to about $500 \mathrm{mg} /$ day of combined EPA and DHA intake for individuals without underlying overt CVD [27].

Given the low prevalence of CVD in populations such as Japanese whose diet is largely based on fish, dietary modification towards greater fish consumption is desirable. However, when adherence to the recommended diet regimen is inadequate or availability of the recommended fish species is limited, it might be necessary to administer purified supplements [13]. An ongoing debate concerns the $n-6: n-3$ ratio. Based on in vitro competition mechanisms, reducing the ratio should in theory lessen the incidence of degenerative diseases, including those with an inflammatory component. However, validity of this hypothesis in vivo has been questioned [17]. Both n-3 and n-6 PUFA are essential. From a nutritional viewpoint, the absolute ratio might not be a useful concept because at any ratio, both intakes could be deficient [40]. It might be better to consider PUFA individually rather than as a ratio (which does not distinguish between ALA and EPA+DHA) and to provide evidence-based dietary advice to increase n-3 PUFA intake rather than just decreasing n-6 PUFA. Assessment of individual needs may be based on the $n-3$ red blood cell index [26], but further studies are needed to validate this novel biomarker for cardiovascular risk.

In spite of the pitfalls of meta-analyses, epidemiological studies have suggested the potential benefits of $n-3$ PUFA on cardiovascular health. Randomized controlled intervention trials continue to show the success of n-3 PUPA in primary and secondary CVD prevention, while evidence from cellular and molecular research indicates that the cardioprotective effects result from a synergism between multiple intricate mechanisms that involve antiinflammation, antiatherosclerotic and antiarrhythmic effects.

Although considerable research ranging from in vitro to animal to human studies has been dedicated to elucidate the activities of $n$-3 PUFA on the cardiovascular system, due to practical and financial reasons, most investigations did not measure PUFA levels in plasma or in red blood cells before and after intervention. This limits the establishment of dose-dependent correlations between plasma PUFA variations (a reliable marker of intake) and clinical outcomes [40].

Generally, n-3 PUFA supplementation has few side effects and is recognized as safe by the US Food and Drug Administration. The target consumption range appears to be $1000 \mathrm{mg} /$ day for patients with CHD or heart failure, but further 
clinical studies are needed to determine optimal dosing for various populations and the relative ratio of DHA to EPA that can provide maximum protection for those at risk of CVD and in the treatment of CVD related disorders. High quality randomized controlled trials are also needed to clarify the benefits of ALA on cardiovascular health. It is unclear whether ALA prevents recurrent coronary events in view of the few studies available. Regardless of the scientific evidence, for people who still wish to supplement their diet with fish oil capsules, the advantage is the virtually lack of any deleterious and side effects of n-3 PUFA and the possibility to find EPA/DHA as oral preparations without prescription at a relatively low cost.

In conclusion, more research is required to clarify the role of $n$-3 PUFA supplementation in primary prevention of CVD. The dose-dependent benefits should be investigated among patients receiving optimal medical therapy for cardiovascular protection, including background use of statins, ACE inhibitors, beta-blockers and aspirin. ${ }^{42}$ Further research is also warranted to provide recommendations to other patient groups (eg diabetes). Nevertheless, given the public health focus on the epidemic of CVD, there is certainly scope for the inclusion of n-3 PUFA therapy as part of a comprehensive CVD management package. It should also be noted that the diverse biological roles played by $n-3$ PUFA extend beyond the cardiovascular system. It is envisaged that further evidence-based recommendations will be issued in the near future.

\section{REFERENCES}

[1]. World Health Organization. Cardiovascular dise-ases (CVDs). Available from: http://www.who.int/ mediacentre/factsheets/fs317/en/index.html

[2]. Centers for Disease Control and Prevention. Heart Disease and Stroke Prevention. Addressing the nation's leading killers: at a glance 2010. Available from: http://www.cdc.gov/chronicdisease/ reso urces/publications/AAG/dhdsp.htm

[3]. National Heart Foundation of Australia. Data and statistics. Available from: http:// www.heartfoundation.org.au/information-forprofessionals/ data-and-statistics.

[4]. Mozaffarian D. JELIS, fish oil and cardiac events. Lancet. 2007;369(9567):1062-1063.

[5]. Meyer BJ. Are we consuming enough long chain o mega-3 polyunsaturated fatty acids for optimal health? Prostaglandins Leukot Essent Fatty Acids. 2011;DOI: 10.1016/j.plefa.2011.04.010
[6]. Chan EJ, Cho L. What can we expect from omega-3 fatty acids? Cleve Clin J Med. 2009;76(4):245-251.

[7]. Russo GL. Dietary $n-6$ and $n-3$ polyunsaturatedfatty acids: from biochemistry to clinical implications in cardiovascular prevention. Biochem Pharmacol. 2009;77(6):937-946.

[8]. Stillwell W, Wassall SR. Docosahexaenoic acid: membrane properties of a unique fatty acid. Chem Phys Lipids. 2003;126(1):1-27.

[9]. Innis SM. Perinatal biochemistry and physiology of long-chain polyunsaturated fatty acids. J Pediatr. 2003;143(4 Suppl):S1-S8.

[10]. Kalmijin S. Fatty acid intake and the risk of dementia and cognitive decline: a review of clinical and epidemiological studies. J Nutr Health Aging. 2000;4(4):202-207.

[11]. Sampath H, Ntambi JM. Polyunsaturated fatty acid regulation of gene expression. Nutr Rev. 2004;62(9):333-339.

[12]. International Society for the Study of Fatty Acids and Lipids. Recommendations for intake of polyunsaturated fatty acids in healthy adults. Available from: http://www.issfal.org

[13], Calzolari I, Fumagalli S, M archionni N, Di Bari M. Polyunsaturated fatty acids and cardiovascular disease. Curr Pharm Des. 2009;15(36):4094-4102.

[14]. Jeppesen PB, Hoy CE, M ortensen PB. Essential fatty acid deficiency in patients receiving home parenteral nutrition. Am J Clin Nutr. 1998;68(1):126133.

[15]. Lafourcade M, Larrieu T, Mato S, et al. Nutritional omega-3 deficiency abolishes endocannabinoidmediated neuronal functions. Nat Neurosci. 2011;14(3):345-350.

[16]. Okuyama H. High n-6 to $n-3$ ratio of dietary fatty acids rather than serum cholesterol as a major risk factor for coronary heart disease. Eur J Lipid Sci Technol. 2001;103(6):418-422.

[17]. Griffin BA. How relevant is the ratio of dietary $n-6$ to n-3 polyunsaturated fatty acids to cardiovascular disease risk? Evidence from the OPTILIP study. Curr Opin Lipidol. 2008;19(1):57-62.

[18]. Tribole EF, Thompson RL, Harrison RA, et al. Excess omega- 6 fats thwart health benefits from omega-3 fats. BM J. 2006;332(7544):752-760.

[19]. Adkins Y, Kelley DS. Mechanisms underlying the cardioprotective effects of omega-3 polyunsaturated fatty acids. J Nutr Biochem. 2010;21(9):781-792.

[20]. Willett WC. The role of dietary $n-6$ fatty acids in the prevention of cardiovascular disease. J Cardiovasc Med. (Hagerstown). 2007;8 Suppl 1:S42-S45.

[21]. Mozaffarian D, Ascherio A, Hu FB, et al. Interplay between different polyunsaturated fatty acids and risk of coronary heart disease in men. Circulation. 2005;111(2):157-164.

[22]. Japanese M inistry of Health, Labour and Welfare. Dietary Reference Intakes for Japanese (2010edition). Available from: http:// www.mhlw.go.jp / bunya/kenkou/sessyu-kijun.html

[23].Ambring A, Johansson M, Axeisen M, Gan L, Strandvik $B$, Friberg P. Mediterranean-inspired diet lowers the ratio of serum phospholipid $n-6$ to $n-3$ fatty acids, the number of leukocytes and platelets, and vascular endothelial growth factor in healthy subjects. Am J Clin Nutr. 2006;83(3):575-581. 


\section{B. Raghu. Prevention of Cardiovascular Disease by Dietary n-3 Fatty Acid .}

[24]. Iso H, Kobayashi M, Ishihara J, et al. Intake of fish and $n 3$ fatty acids and risk of coronary heart disease among Japanese: the Japan Public Health CenterBased (JPHC) Study Cohort I. Circulation. 2006; 113(2):195-202.

[25]. Harris WS. The omega-3 index as a risk factor for coronary heart disease. Am J Clin Nutr. 2008;87(6):1997S-2002S.

[26]. Harris WS. The omega-3 index: from biomarker to risk marker to risk factor. Curr Atheroscler Rep. 2009;11(6):411-417.

[27]. Lavie C], M ilani RV, Mehra MR, Ventura HO. Omega3 polyunsaturated fatty acids and cardiovascular diseases. J Am Coll Cardiol. 2009;54(7):585-594.

[28]. Patel JV, Tracey I, Hughes EA, Lip GY. Omega-3 polyunsaturated fatty acids: a necessity for a comprehensive secondary prevention strategy. Vasc Health Risk M anag. 2009;5:801-810.

[29]. Yamagishi K, Iso H, Date C, et al. Fish, omega-3 polyunsaturated fatty acids, and mortality from cardiovascular diseases in a nationwide community-based cohort of Japanese men and women the JACC (Japan Collaborative Cohort Study for Evaluation of Cancer Risk) Study. J Am Coll Cardiol. 2008;52(12):988-996.

[30]. Hooper L, Thompson RL, Harrison R A, et al. Omega 3 fatty acids for prevention and treatment of cardiovascular disease. Cochrane Database Syst Rev. 2004;18(4):CD003177.

[31]. Studer M, Briel M, Leimenstoll B, Glass TR, Bucher HC. Effect of different antilipidemic agents and diets on mortality: a systematic review. Arch Intern Med. 2005;165(7):725-730.

[32]. Wang $C$, Harris WS, Chung M, et al. n-3 Fatty acids from fish or fish-oil supplements, but not alphalinolenic acid, benefit cardiovascular disease outcomes in primary- and secondary-prevention studies: a systematic review. Am J Clin Nutr. 2006;84(1):5-17.

[33]. Balk EM, Lichtenstein AH, Chung M, Kupelnick B, Chew $P$, Lau J. Effects of omega-3 fatty acids on coronary restenosis, intima-media thickness, and exercise tolerance: A systematic review. Atherosclerosis. 2006;184(2):237-246.

[34]. Balk EM, Lichtenstein AH, Chung M, Kupelnick B, Chew $P$, Lau J. Effects of omega-3 fatty acids on serum markers of cardiovascular disease risk: a systematic review. Atherosclerosis. 2006;189(1):19-30.

[35]. Castro IA, Barroso LP, Sinnecker P. Functional foods for coronary heart disease risk reduction: a metaanalysis using a multivariate approach. Am J Clin Nutr. 2005;82(1):32-40.

[36]. Eslick GD, Howe PRC, Smith C, Priest R, Bensoussan A. Benefits of fish oil supplementation in hyperlipidemia: a systematic review and metaanalysis. Int J Cardiol. 2009;136(1):4-16.

[37]. Lee KW, Lip GY. Effects of lifestyle on hemostasis, fibrinolysis, and platelet reactivity: a systematic review. Arch Intern M ed. 2003;163(19):2368-2392.
[38]. M ensink RP, Zock PL, Kester AD, Katan M B. Effects of dietary fatty acids and carbohydrates on the ratio of serum total to HDL cholesterol and on serum lipids and apolipoproteins: a meta-analysis of 60 controlled trials. Am J Clin Nutr. 2003;77(5):1146-1155.

[39]. Wendland E, Farmer A, Glasziou P, Neil A. Effect of alpha linolenic acid on cardiovascular risk markers: a systematic review. Heart. 2006;92(2): 166-169.

[40]. Richard D, Bausero P, Schneider C, Visioli F. Polyunsaturated fatty acids and cardiovascular disease. Cell Mol Life Sci. 2009;66(20):3277-3288.

[41]. Massaro M, Scoditti E, Carluccio MA, De Caterina R. Basic mechanisms behind the effects of $n-3$ fatty acids on cardiovascular disease. Prostaglandins Leukot Essent Fatty Acids. 2008;79(3-5):109-115.

[42].Watts GF, M ori TA. Recent advances in understanding the role and use of marine $w 3$ polyunsaturated fatty acids in cardiovascular protection. Curr Opin Lipidol. 2011;22(1): 70-71.

[43]. Kris-Etherton PM , Harris WS, Appel L]; for American Heart Association Nutrition Committee. Fish consumption, fish oil, omega-3 fatty acids, and cardiovascular disease. Circulation. 2002; 106(21):2747-2757.

[44]. National Heart Foundation of Australia. Position statement: Fish, fish oils, n-3 polyunsaturated fatty acids and cardiovascular health. Updated November 2008. Available from: www.heartfoundation.org.au

[45]. National Health and Medical Research Council. Nutrient reference values for Australia and New Zealand including recommended dietary intakes. Canberra: Australian Government Department of Health and Ageing; 2006.

[46]. USDA Agricultural Research Service. Nutrient Data Laboratory. Available from: http:// www.ars.usda.gov/nutrientdata

[47]. Saravanan P, Davidson NC, Schmidt EB, Calder PC. Cardiovascular effects of marine omega-3 fatty acids. Lancet. 2010;376(9740):540-550.

[48]. American Heart Association. Fish and Omega-3 Fatty Acids. Available from: http:// www.amhrt.org/ HEARTORG/GettingHealthy/NutritionCenter/ HealthyDietGoals/Fish-and-Omega-3-FattyAcids_UCM_303248_Article.jsp

[49]. B. Raghhu. and P. Venkatesan. Effect of n-3 fatty acid supplementation on blood glucose, lipid profile and cytokines in humans. A pilot study. Ind. J. Clin. Biochem. 2008;23(1):85-88.

[50]. B.Raghu and P.Venkatesan. Relationship between cigarette smoking and novel risk factors for cardiovascular disease. Journal of Biomedical Sciences. 2012;1:1-4.

How to cite this article:

B. Raghu. Prevention of Cardiovascular Disease by Dietary n-3 Fatty Acid . Int J Intg M ed Sci 2016;3(9):404-411. DOI: 10.16965/ijims.2016.124 